\title{
A Highly Efficient and Accurate Finite Iterative Method for Solving Linear Two-Dimensional Fredholm Fuzzy Integral Equations of the Second Kind Using Triangular Functions
}

\author{
Mohamed A. Ramadan $\left(\mathbb{D}\right.$, Heba S. Osheba ${ }^{D}$, and Adel R. Hadhoud \\ Faculty of Science, Menoufia University, Shebin El-Kom, Egypt \\ Correspondence should be addressed to Mohamed A. Ramadan; ramadanmohamed13@yahoo.com
}

Received 12 April 2020; Revised 12 June 2020; Accepted 3 August 2020; Published 29 August 2020

Academic Editor: Praveen Agarwal

Copyright ( 2020 Mohamed A. Ramadan et al. This is an open access article distributed under the Creative Commons Attribution License, which permits unrestricted use, distribution, and reproduction in any medium, provided the original work is properly cited.

\begin{abstract}
This work introduces a computational method for solving the linear two-dimensional fuzzy Fredholm integral equation of the second form (2D-FFIE-2) based on triangular basis functions. We have used the parametric form of fuzzy functions and transformed a 2D-FFIE-2 with three variables in crisp case to a linear Fredholm integral equation of the second kind. First, a method based on the use of two $m$-sets of orthogonal functions of triangular form is implemented on the integral equation under study to be changed to coupled algebraic equation system. In order to solve these two schemes, a finite iterative algorithm is then applied to evaluate the coefficients that provided the approximate solution of the integral problems. Three examples are given to clarify the efficiency and accuracy of the method. The obtained numerical results are compared with other direct and exact solutions.
\end{abstract}

\section{Introduction}

Several methods have been developed to estimate the solution of integral equation systems [1-3]. Many simple functions are used to approximate the solution of integral equations, such as orthogonal bases dependent on wavelets [4]. In addition, Maleknejad and Mirzaee developed the rationalized Haar functions [5] to approximate the solutions of the Fredholm linear integral equation method. In addition, second-type Fredholm integral equations are solved using direct triangular functions method as seen in [6] and using iterative algorithm-hybrid triangular functions method presented by Ramadan and Ali [7] where this hybrid method treats Fredholm integral equation of one dimension. More recently, Ramadan et al. [8] implemented such hybrid method to tackle system of two linear Fredholm integral equations of one dimension.

Furthermore, Maleknejad et al. [9] suggested by block pulse functions a numerical solution of the integral secondtype equation.
It is explained using a series of orthogonal triangular functions, derived from the series of block pulses. Nevertheless, the fuzzy integral equations (FIEs) are required to solve and research a wide number of problems in various applied mathematics subjects, such as connection to physics, spatial, medical, and biology. FIEs therefore require approximate numerical solutions, as they are typically difficult to analytically solve. This thesis introduces a methodology used by the triangular functions (TFs) to solve the fuzzy linear FIE method of the second kind. In various implementation problems, certain parameters are typically represented by a fuzzy number rather than a crisp state, which involves the creation of mathematical models and computational algorithms to handle and solve the general fuzzy integral equations. A general method for solving the fuzzy Fredholm second-type integral equation is proposed in [10]. Recently, numerical methods have been developed to solve linear fuzzy Fredholm integral equation of the second kind in one-dimensional space (1D-FFIE-2) and two-dimensional space (2D-FFIE-2). Also, Fredholm fuzzy integral equations 
of the second kind are solved using the triangular functions [11], and numerical solution of linear Fredholm fuzzy equation of the second kind by block pulse functions is considered in [12]. Barkhordary et al. and Ramadan et al. $[13,14]$ presented a numerical technique for solving the fuzzy Fredholm integral equation of second kind. Numerical solution of two-dimensional fuzzy Fredholm integral equations of the second kind is presented via direct method using triangular functions [15]. Nouriani et al. [16] proposed a quadrature iterative method for solving the two-dimensional fuzzy Fredholm integral equations. Ezzati and Ziari [17], Hengamian Asl and Saberi-Nadjafi [18], and Bica and Popescu [19] illustrated a solution of the two-dimensional fuzzy Fredholm integral equations. A modified homotopy perturbation method for solving the two-dimensional fuzzy Fredholm integral equation is detailed in [20]. A two-dimensional nonlinear Volterra-Fredholm fuzzy integral equation is solved by using the Adomian decomposition method [21] and fuzzy bivariate triangular functions [22].

The aim of paper is to generalize the work proposed in [7] and [8] of these basis orthogonal triangular functions on $(0,1)$ to solve two-dimensional fuzzy Fredholm integral equations.

$$
\widetilde{u}(x, y)=\widetilde{f}(x, y)+\lambda \int_{a}^{b} \int_{c}^{d} k(x, y, s, t) \widetilde{u}(s, t) \mathrm{d} s \mathrm{~d} t .
$$

Section 2 presents some definitions and properties of the orthogonal triangular functions (TFs) (1D-TFs and 2D-TFs). Also, it expands functions by TFs. In Section 3, the definitions and properties of fuzzy function are given while a finite iterative algorithm is presented to solve coupled system of matrix equations in Section 4. The two-dimensional fuzzy integral equation is demonstrated and explained in Section 5 while the suggested method and the proposed iterative algorithm are detailed in Section 6. The illustrative examples and numerical results obtained are presented and discussed in Section 8.

\section{Review of Triangular Functions (TFs)}

\subsection{Triangular Functions (TFs) of One Dimension}

Definition 1. Two $m$-sets of triangular functions (TFs) are defined over the interval $[0, T)[5]$ :

$$
\begin{aligned}
& T 1_{i}(t)= \begin{cases}1-\frac{t-i h}{h}, & i h \leq t<(1+i) h, \\
0, & \text { o.w, }\end{cases} \\
& T 2_{i}(t)= \begin{cases}\frac{t-i h}{h}, & i h \leq t<(1+i) h, \\
0, & \text { o.w, }\end{cases}
\end{aligned}
$$

where $i=0,1, \ldots, m-1 ; m$ has a positive integer value; $h=(T / m) ; T 1_{i}$ is the $i$ th left-handed triangular function; and $T 2_{i}$ is the $i$ th right-handed triangular function.
Assuming $T=1$, the TFs are defined over $[0,1)$ and $h=(1 / m)$. Based on this definition, it is clear that TFs are disjoint, orthogonal, and complete [5]. Therefore, one may write

$$
\begin{aligned}
& \int_{0}^{1} T 1_{i}(t) T 1_{j}(t) \mathrm{d} t=\int_{0}^{1} T 2_{i}(t) T 2_{j}(t) \mathrm{d} t= \begin{cases}\frac{h}{3}, & i=j, \\
0, & i \neq j,\end{cases} \\
& \int_{0}^{1} T 1_{i}(t) T 2_{j}(t) \mathrm{d} t=\int_{0}^{1} T 2_{i}(t) T 1_{j}(t) \mathrm{d} t= \begin{cases}\frac{h}{6}, & i=j, \\
0, & i \neq j .\end{cases}
\end{aligned}
$$

The first $m$ terms in the left-hand triangular functions and in the right-hand triangular functions can be written concisely in $m$-vectors format as

$$
\begin{aligned}
T 1(t) & =\left[T 1_{0}(t), T 1_{1}(t), \ldots, T 1_{m-1}(t)\right]^{T}, \\
T 2(t) & =\left[T 2_{0}(t), T 2_{1}(t), \ldots, T 2_{m-1}(t)\right]^{T},
\end{aligned}
$$

where $T 1(t)$ and $T 2(t)$ are called left-handed triangular function (LHTF) vector and right-handed triangular function (RHTF) vector, respectively. The product of two TF vectors yields the following properties:

$$
\begin{gathered}
T 1(t) T 1^{T}(t) \cong\left(\begin{array}{cccc}
T 1_{0}(t) & 0 & \cdots & 0 \\
0 & T 1_{1}(t) & \cdots & 0 \\
\vdots & \vdots & \ddots & \vdots \\
0 & 0 & 0 & T 1_{m-1}(t)
\end{array}\right), \\
T 2(t) T 2^{T}(t) \cong\left(\begin{array}{cccc}
T 2_{0}(t) & 0 & \cdots & 0 \\
0 & T 2_{1}(t) & \cdots & 0 \\
\vdots & \vdots & \ddots & \vdots \\
0 & 0 & 0 & T 2_{m-1}(t)
\end{array}\right),
\end{gathered}
$$

$$
\begin{aligned}
& T 1(t) T 2^{T}(t) \cong 0, \\
& T 2(t) T 1^{T}(t) \cong 0,
\end{aligned}
$$

where 0 is the zero $m \times m$ matrix. Also,

$$
\begin{aligned}
& \int_{0}^{1} T 1(t) T 1^{T}(t) \mathrm{d} t=\int_{0}^{1} T 2(t) T 2^{T}(t) \mathrm{d} t \cong \frac{h}{3} I, \\
& \int_{0}^{1} T 1(t) T 2^{T}(t) \mathrm{d} t=\int_{0}^{1} T 2(t) T 1^{T}(t) \mathrm{d} t \cong \frac{h}{6} I,
\end{aligned}
$$

in which $I$ is an $m \times m$ identity matrix.

2.2. Two-Dimensional Triangular Functions and Their Properties [15]. An $\left(m_{1} \times m_{2}\right)$-set of $2 \mathrm{D}-\mathrm{TFs}$ on the region $(\Omega=$ $[0,1] \times[0.1])$ is defined by 


$$
T_{i, j}^{1,1}(s, t)=\left\{\begin{array}{ll}
\left(1-\frac{s-i h_{1}}{h_{1}}\right)\left(1-\frac{t-j h_{2}}{h_{2}}\right), & \begin{array}{l}
i h_{1} \leq s \leq(i+1) h_{1}, \\
j h_{2} \leq t \leq(j+1) h_{2},
\end{array} \\
0, & \text { otherwise, }
\end{array} \quad \begin{array}{l}
T_{i, j}^{1,1}(s, t)+T_{i, j}^{1,2}(s, t)+T_{i, j}^{2,1}(s, t)+T_{i, j}^{2,2}(s, t)=\phi_{i, j}(s, t), \\
(10)
\end{array}\right.
$$$$
T_{i, j}^{1,2}(s, t)= \begin{cases}\left(1-\frac{s-i h_{1}}{h_{1}}\right)\left(\frac{t-j h_{2}}{h_{2}}\right), & i h_{1} \leq s \leq(i+1) h_{1}, \\ 0, & j h_{2} \leq t \leq(j+1) h_{2}, \\ & \text { otherwise, }\end{cases}
$$$$
T_{i, j}^{2,1}(s, t)= \begin{cases}\left(\frac{s-i h_{1}}{h_{1}}\right)\left(1-\frac{t-j h_{2}}{h_{2}}\right), & i h_{1} \leq s \leq(i+1) h_{1}, \\ & j h_{2} \leq t \leq(j+1) h_{2}, \\ 0, & \text { otherwise, }\end{cases}
$$$$
T_{i, j}^{2,2}(s, t)= \begin{cases}\left(\frac{s-i h_{1}}{h_{1}}\right)\left(\frac{t-j h_{2}}{h_{2}}\right), & i h_{1} \leq s \leq(i+1) h_{1}, \\ & j h_{2} \leq t \leq(j+1) h_{2}, \\ 0, & \text { otherwise, }\end{cases}
$$

where: $\quad i=0,1,2, \ldots ; m_{1}-1 ; j=0,1,2, \ldots, m_{2}-1$; $h_{1}=\left(1 / m_{1}\right) ; h_{2}=\left(1 / m_{2}\right) ;$ and $m_{1}$ and $m_{2}$ are arbitrary positive integers. Therefore,

$$
\left\{\begin{array}{l}
T_{i, j}^{1,1}(s, t)=T 1_{i}(s) \cdot T 1_{j}(t), \\
T_{i, j}^{1,2}(s, t)=T 1_{i}(s) \cdot T 2_{j}(t), \\
T_{i, j}^{2,1}(s, t)=T 2_{i}(s) \cdot T 1_{j}(t), \\
T_{i, j}^{2,2}(s, t)=T 2_{i}(s) \cdot T 2_{j}(t) .
\end{array}\right.
$$

where $\varphi_{i, j}(s, t)$ is the $\left\{i m_{2}+j+1\right\}$ th block pulse function defined on $i h_{1} \leq s \leq(i+1) h_{1}$ and $j h_{2} \leq t \leq(j+1) h_{2}$ as

$$
\varphi_{i, j}(s, t)= \begin{cases}1, & i h_{1} \leq s \leq(i+1) h_{1}, \\ & j h_{2} \leq t \leq(j+1) h_{2}, \\ 0, & \text { otherwise. }\end{cases}
$$

Each of the sets $\left\{T_{i, j}^{1,1}(s, t), T_{i, j}^{1,2}(s, t), T_{i, j}^{2,1}(s, t)\right\}$ and $\left\{T_{i, j}^{2,2}(s, t)\right\}$ is obviously disjoint:

$$
T_{i_{1} j_{1}}^{p_{1}, q_{1}}(s, t) \cdot T_{i_{2} j_{2}}^{p_{2}, q_{2}}(s, t) \approx \begin{cases}T_{i_{1} j_{1}}^{p_{1}, q_{1}}(s, t), & p_{1}=p_{2}, q_{1}=q_{2}, \\ 0, & i_{1}=i_{2}, j_{1}=j_{2}, \\ 0, & \text { otherwise. }\end{cases}
$$

For $p, q \in\{1,2\}, \quad i_{1}, i_{2}=0,1,2, \ldots, m_{1}-1 \quad$ and $j_{1}, j_{2}=0,1,2, \ldots, m_{2}-1$.

Also, the 2D-TFs are orthogonal, that is,

$$
\int_{0}^{1} \int_{0}^{1} T_{i_{1} j_{1}}^{p_{1}, q_{1}}(s, t) \cdot T_{i_{2} j_{2}}^{p_{2}, q_{2}}(s, t) \mathrm{d} s \mathrm{~d} t=\Delta_{p_{1}, p_{2}} \delta_{i_{1}, i_{2}} \cdot \Delta_{q_{1}, q_{2}} \delta_{j_{1}, j_{2}},
$$

where $\delta$ denotes the Kronecker delta function and

$$
\Delta_{\alpha, \beta}= \begin{cases}\frac{h}{3}, & \alpha=\beta \in\{1,2\} \\ \frac{h}{6}, & \alpha \neq \beta\end{cases}
$$

On the other hand, if

$$
\begin{aligned}
& T 11(s, t)=\left[T_{0,0}^{1,1}(s, t), \ldots, T_{i, m_{2}-1}^{1,1}(s, t), T_{1,0}^{1,1}(s, t), \ldots, T_{m_{1}-1, m_{2}-1}^{1,1}(s, t)\right]^{T}, \\
& T 12(s, t)=\left[T_{0,0}^{1,2}(s, t), \ldots, T_{i, m_{2}-1}^{1,2}(s, t), T_{1,0}^{1,2}(s, t), \ldots, T_{m_{1}-1, m_{2}-1}^{1,2}(s, t)\right]^{T}, \\
& T 21(s, t)=\left[T_{0,0}^{2,1}(s, t), \ldots, T_{i, m_{2}-1}^{2,1}(s, t), T_{1,0}^{2,1}(s, t), \ldots, T_{m_{1}-1, m_{2}-1}^{2,1}(s, t)\right]^{T}, \\
& T 22(s, t)=\left[T_{0,0}^{2,2}(s, t), \ldots, T_{i, m_{2}-1}^{2,2}(s, t), T_{1,0}^{2,2}(s, t), \ldots, T_{m_{1}-1, m_{2}-1}^{2,2}(s, t)\right]^{T},
\end{aligned}
$$

then $T(s, t)$, the 2D-TF vector, can be defined as 
$\begin{aligned} & T(s, t)=\left[\begin{array}{c}T 11(s, t) \\ T 12(s, t) \\ T 21(s, t) \\ T 22(s, t)\end{array}\right]_{4 m_{1} m_{2} \times 1}, \\ & T 11 \cdot T 11^{T} \simeq\left[\begin{array}{cccc}T_{0,0}^{1,1} & 0 & \cdots & 0 \\ 0 & T_{0,1}^{1,1} & \ddots & \vdots \\ \vdots & \vdots & \ddots & 0 \\ 0 & 0 & 0 & T_{m_{1}-1, m_{2}-1}^{1,1}\end{array}\right]=\operatorname{diag}(T 11),\end{aligned}$

$$
\begin{aligned}
& T 11 \cdot T 12^{T} \simeq 0_{m_{1} m_{2} \times m_{1} m_{2}}, \\
& T 11 \cdot T 21^{T} \simeq 0_{m_{1} m_{2} \times m_{1} m_{2}}, \\
& T 11 \cdot T 22^{T} \simeq 0_{m_{1} m_{2} \times m_{1} m_{2}} .
\end{aligned}
$$

These relations are also satisfied for $T 12(s, t), T 21(s, t), T 22(s, t)$, similarly. Hence,

$$
T \cdot T^{T} \simeq\left[\begin{array}{llll}
\operatorname{diag}(T 11) & 0_{m_{1} m_{2} \times m_{1} m_{2}} & 0_{m_{1} m_{2} \times m_{1} m_{2}} & 0_{m_{1} m_{2} \times m_{1} m_{2}} \\
0_{m_{1} m_{2} \times m_{1} m_{2}} & \operatorname{diag}(T 12) & 0_{m_{1} m_{2} \times m_{1} m_{2}} & 0_{m_{1} m_{2} \times m_{1} m_{2}} \\
0_{m_{1} m_{2} \times m_{1} m_{2}} & 0_{m_{1} m_{2} \times m_{1} m_{2}} & \operatorname{diag}(T 21) & 0_{m_{1} m_{2} \times m_{1} m_{2}} \\
0_{m_{1} m_{2} \times m_{1} m_{2}} & 0_{m_{1} m_{2} \times m_{1} m_{2}} & 0_{m_{1} m_{2} \times m_{1} m_{2}} & \operatorname{diag}(T 22)
\end{array}\right],
$$

$\operatorname{or} T(s, t) \cdot T^{T}(s, t) \simeq \operatorname{diag}(T(s, t))$.

Finally by the orthogonality of $T 11$, we have

$$
\begin{aligned}
& \int_{0}^{1} \int_{0}^{1} T 11(s, t)^{T} T 11(s, t) \mathrm{d} s \mathrm{~d} t=\frac{h_{1}}{3} I_{m_{1} \times m_{1}} \otimes \frac{h_{2}}{3} I_{m_{2} \times m_{2},}, \\
& \int_{0}^{1} \int_{0}^{1} T 11(s, t)^{T} T 12(s, t) \mathrm{d} s \mathrm{~d} t=\frac{h_{1}}{3} I_{m_{1} \times m_{1}} \otimes \frac{h_{2}}{6} I_{m_{2} \times m_{2},}, \\
& \int_{0}^{1} \int_{0}^{1} T 11(s, t)^{T} T 21(s, t) \mathrm{d} s \mathrm{~d} t=\frac{h_{1}}{6} I_{m_{1} \times m_{1}} \otimes \frac{h_{2}}{3} I_{m_{2} \times m_{2},}, \\
& \int_{0}^{1} \int_{0}^{1} T 11(s, t)^{T} T 22(s, t) \mathrm{d} s \mathrm{~d} t=\frac{h_{1}}{6} I_{m_{1} \times m_{1}} \otimes \frac{h_{2}}{6} I_{m_{2} \times m_{2}},
\end{aligned}
$$

where $\otimes$ denotes the Kronecker product defined for two arbitrary matrices $P$ and $Q$ as

$$
P \otimes Q=P_{i, j} Q .
$$

The same equations are implied for $T 12(s, t), T 21(s, t)$, and $T 22(s, t)$, by similar computations. Hence, we can carry out double integration of $T(s, t)$ :

$$
\int_{0}^{1} \int_{0}^{1} T(s, t)^{T} T(s, t) \mathrm{d} s \mathrm{~d} t=D,
$$

where $D$ is $4 m_{1} m_{2} \times 4 m_{1} m_{2}$ matrix as follows:

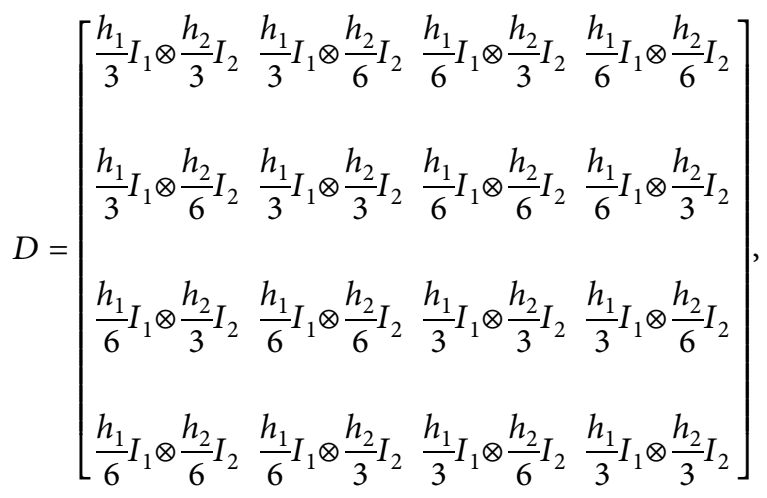

where $I_{1}=I_{m_{1} \times m_{1}}$ and $I_{2}=I_{m_{2} \times m_{2}}$.

2.3. Function Expansion with $1 D-T F s$ and $2 D-T F s$. The expansion of functions using triangular functions occurs in four situations.

(1) The expansion of function $f(t)$ over $[0,1)$ with respect to $1 \mathrm{D}$-TFs is compactly written as

$$
f(t) \cong \sum_{i=0}^{m-1} c_{i} T 1_{i}(t)+\sum_{i=0}^{m-1} d_{i} T 2_{i}(t)=c^{T} T 1(t)+d^{T} T 2(t),
$$

where we may put $c_{i}=f(i h)$ and $d_{i}=f((i+1) h)$ for $i=0,1, \ldots, m-1$. 
(2) The expansion of the function $f(s, t)$ defined over $\Omega$ $([0,1) \times[0,1))$ by $2 \mathrm{D}-\mathrm{TF}$ is as follows:

$$
\begin{aligned}
& f(s, t)=\sum_{0}^{m_{1}-1} \sum_{0}^{m_{2}-1} c_{i, j} T_{i, j}^{1,1}(s, t)+\sum_{i=0}^{m_{1}-1} \sum_{j=0}^{m_{2}-1} d_{i, j} T_{i, j}^{1,2}(s, t)+\sum_{i=0}^{m_{1}-1} \sum_{j=0}^{m_{2}-1} e_{i, j} T_{i, j}^{2,1}(s, t)+\sum_{i=0}^{m_{1}-1} \sum_{j=0}^{m_{2}-1} l_{i, j} T_{i, j}^{2,2}(s, t) \\
& f(s, t)=C^{T} T 11(s, t)+D^{T} T 12(s, t)+E^{T} T 21(s, t)+L^{T} T 22(s, t)
\end{aligned}
$$

where $F$ is a $4 m_{1} m_{2}$ vector given by

$$
F=\left[\begin{array}{llll}
C^{T} & D^{T} & E^{T} & L^{T}
\end{array}\right]^{T},
$$

and $T(s, t)$ is defined in equation (21). The $2 \mathrm{D}-\mathrm{TF}$ coefficients in $C, D, E$, and $L$ can be computed by sampling the function $f(s, t)$ at grid points $s_{i}$ and $t_{j}$ such that $s_{i}=i h_{1}$ and $t_{j}=j h_{2}$, for various $i$ and $j$. So, we have

$$
\begin{aligned}
& C_{k}=c_{i, j}=f\left(s_{i}, t_{j}\right), \\
& D_{k}=d_{i, j}=f\left(s_{i}, t_{j+1}\right), \\
& E_{k}=e_{i, j}=f\left(s_{i+1}, t_{j}\right), \\
& L_{k}=l_{i, j}=f\left(s_{i+1}, t_{j+1}\right),
\end{aligned}
$$

where $k=i m_{2}+j$ and $i=0,1,2, \ldots, m_{1}-1$, $j=0,1,2, \ldots, m_{2}-1$. The $4 m_{1} m_{2}$ vector $F$ is called the $2 \mathrm{D}-\mathrm{TF}$ coefficient vector.

(3) The expansion of the function $f(s, t, r)$ of three variables on $(\Omega \times[0,1])$ with respect to $2 \mathrm{D}$-TFs and $1 \mathrm{D}-\mathrm{TFs}$ is as follows:

$$
f(s, t, r)=T^{T}(s, t) \cdot F \cdot T(r),
$$

where $T(s, t)$ and $T(r)$ are $2 \mathrm{D}-\mathrm{TF}$ vector and $1 \mathrm{D}-\mathrm{TF}$ vector of dimension $4 m_{1} m_{2}$ and $2 m_{3}$, respectively, and $F$ is a $\left(4 m_{1} m_{2} \times 2 m_{3}\right) 2 \mathrm{D}$-TF coefficient matrix. This matrix can be represented as

$$
F=\left[\begin{array}{ll}
F 11 & F 12 \\
F 21 & F 22 \\
F 31 & F 32 \\
F 41 & F 42
\end{array}\right],
$$

where each block of $F$ is an $\left(m_{1} m_{2} \times m_{3}\right)$-matrix that can be computed by sampling the function $f(s, t, r)$ at grid points $\left(s_{i}, t_{j}, r_{k}\right)$ such that

$$
\begin{aligned}
& s_{i}=i h_{1}, \quad i=0,1, \ldots, m_{1}-1, h_{1}=\frac{1}{m_{1}}, \\
& t_{j}=j h_{2}, \quad j=0,1, \ldots, m_{2}-1, h_{2}=\frac{1}{m_{2}}, \\
& r_{k}=k h_{3}, \quad k=0,1, \ldots, m_{3}-1, h_{3}=\frac{1}{m_{3}} .
\end{aligned}
$$

Let $l=i m_{2}+j$; then,

$$
\begin{aligned}
& F 11_{l, k}=f\left(s_{i}, t_{j}, r_{k}\right), \\
& F 12_{l, k}=f\left(s_{i}, t_{j}, r_{k+1}\right), \\
& F 21_{l, k}=f\left(s_{i}, t_{j+1}, r_{k}\right), \\
& F 22_{l, k}=f\left(s_{i}, t_{j+1}, r_{k+1}\right), \\
& F 21_{l, k}=f\left(s_{i}, t_{j+1}, r_{k}\right), \\
& F 22_{l, k}=f\left(s_{i}, t_{j+1}, r_{k+1}\right), \\
& F 31_{l, k}=f\left(s_{i+1}, t_{j}, r_{k}\right), \\
& F 32_{l, k}=f\left(s_{i+1}, t_{j}, r_{k+1}\right), \\
& F 31_{l, k}=f\left(s_{i+1}, t_{j}, r_{k}\right), \\
& F 32_{l, k}=f\left(s_{i+1}, t_{j}, r_{k+1}\right), \\
& F 41_{l, k}=f\left(s_{i+1}, t_{j+1}, r_{k}\right), \\
& F 42_{l, k}=f\left(s_{i+1}, t_{j+1}, r_{k+1}\right) .
\end{aligned}
$$

(4) The expansion of the function $k(s, t, x, y)$ of four variables on $(\Omega \times \Omega)$ with respect to $2 \mathrm{D}$-TFs is as follows:

$$
k(s, t, x, y)=T^{T}(s, t) \cdot K \cdot T(x, y),
$$

where $T(S, T)$ and $T(x, y)$ are $2 \mathrm{D}$-TF vectors of dimension $4 m_{1} m_{2}$ and $4 m_{3} m_{4}$, respectively, and $K$ is a $\left(4 m_{1} m_{2} \times 4 m_{3} m_{4}\right) 2 \mathrm{D}$-TF coefficient matrix. This matrix can be represented as 


$$
K=\left[\begin{array}{llll}
K 11 & K 12 & K 13 & K 14 \\
K 21 & K 22 & K 23 & K 24 \\
K 31 & K 32 & K 33 & K 34 \\
K 41 & K 42 & K 43 & K 44
\end{array}\right],
$$

where each block of $K$ is an $\left(m_{1} m_{2} \times m_{3} m_{4}\right)$ matrix that can be computed by sampling the function $k(s, t, x, y)$ at grid points $\left(s_{i_{1}}, t_{j_{1}}, x_{i_{2}}, y_{j_{2}}\right)$ such that

$$
\begin{aligned}
& s_{i_{1}}=i_{1} h_{1}, \quad i_{1}=0,1, \ldots, m_{1}-1, h_{1}=\frac{1}{m_{1}}, \\
& t_{j_{1}}=j_{1} h_{2}, \quad j_{1}=0,1, \ldots, m_{2}-1, h_{2}=\frac{1}{m_{2}}, \\
& x_{i_{2}}=i_{2} h_{3}, \quad i_{2}=0,1, \ldots, m_{3}-1, h_{3}=\frac{1}{m_{3}}, \\
& y_{j_{2}}=j_{2} h_{3}, \quad j_{2}=0,1, \ldots, m_{4}-1, h_{4}=\frac{1}{m_{4}} .
\end{aligned}
$$

Let $p=i_{1} m_{2}+j_{1}$ and $q=i_{2} m_{4}+j_{2}$; then,

$$
\begin{aligned}
& K 11_{p, q}=k\left(s_{i_{1}}, t_{j_{1}}, x_{i_{2}}, y_{j_{2}}\right), \\
& K 12_{p, q}=k\left(s_{i_{1}}, t_{j_{1}}, x_{i_{2}}, y_{j_{2}+1}\right), \\
& K 13_{p, q}=k\left(s_{i_{1}}, t_{j_{1}}, x_{i_{2}+1}, y_{j_{2}}\right), \\
& K 14_{p, q}=k\left(s_{i_{1}}, t_{j_{1}}, x_{i_{2}+1}, y_{j_{2}+1}\right), \\
& K 21_{p, q}=k\left(s_{i_{1}}, t_{j_{1}+1}, x_{i_{2}}, y_{j_{2}}\right), \\
& K 22_{p, q}=k\left(s_{i_{1}}, t_{j_{1}+1}, x_{i_{2}}, y_{j_{2}+1}\right), \\
& K 23_{p, q}=k\left(s_{i_{1}}, t_{j_{1}+1}, x_{i_{2}+1}, y_{j_{2}}\right), \\
& K 24_{p, q}=k\left(s_{i_{1}}, t_{j_{1}+1}, x_{i_{2}+1}, y_{j_{2}+1}\right), \\
& K 31_{p, q}=k\left(s_{i_{1}+1}, t_{j_{1}}, x_{i_{2}}, y_{j_{2}}\right), \\
& K 32_{p, q}=k\left(s_{i_{1}+1}, t_{j_{1}}, x_{i_{2}}, y_{j_{2}+1}\right), \\
& K 33_{p, q}=k\left(s_{i_{1}+1}, t_{j_{1}}, x_{i_{2}+1}, y_{j_{2}}\right), \\
& K 34_{p, q}=k\left(s_{i_{1}+1}, t_{j_{1}}, x_{i_{2}+1}, y_{j_{2}+1}\right), \\
& K 41_{p, q}=k\left(s_{i_{1}+1}, t_{j_{1}+1}, x_{i_{2}}, y_{j_{2}}\right), \\
& K 42_{p, q}=k\left(s_{i_{1}+1}, t_{j_{1}+1}, x_{i_{2}}, y_{j_{2}+1}\right), \\
& K 43_{p, q}=k\left(s_{i_{1}+1}, t_{j_{1}+1}, x_{i_{2}+1}, y_{j_{2}}\right), \\
& K 44_{p, q}=k\left(s_{i_{1}+1}, t_{j_{1}+1}, x_{i_{2}+1}, y_{j_{2}+1}\right) .
\end{aligned}
$$

In this paper, we suppose that $m_{1}=m_{2}=m_{3}=m_{4}=M$ for convergence.

\section{Fuzzy Functions}

We now remember through the paper some definitions that are required.
Definition 2. A fuzzy number is a fuzzy set $\mathrm{u}: R^{1} \longrightarrow[0,1]$ that conforms to the following condition [23]:

(a) $u$ is upper semicontinuous

(b) $u(x)=0$ outside some interval $[c, d]$

(c) There are real numbers $a$ and $b, c \leq a \leq b \leq d$, for which

(i) $u(x)$ is increasing in monotonic manner on $[c$, a]

(ii) $u(x)$ is decreasing in monotonic manner on $[b$, d]

(iii) $u(x)=1$ for $a \leq x \leq b$

Definition 3. A fuzzy number $u$ is a pair $(\underline{u}(r), \bar{u}(r))$ of functions $\underline{u}(r)$ and $\bar{u}(r), 0 \leq r \leq 1$, satisfying the following requirement [5]:

(a) $\underline{u}(r)$ is bounded monotonic increasing left continuous function

(b) $\bar{u}(r)$ is bounded monotonic decreasing left continuous function

(c) $\underline{u}(r) \leq \bar{u}(r), 0 \leq r \leq 1$

For arbitrary $u=(\underline{u}(r), \bar{u}(r)), \quad v=(\underline{v}(r), \bar{v}(r))$, and $k>0$, we define addition $(u+v)$ and multiplication by $k$ as

$$
\begin{aligned}
(\underline{u+v})(r) & =\underline{u}(r)+\underline{v}(r) \\
(\overline{u+v})(r) & =\bar{u}(r)+\bar{v}(r), \\
(\underline{k u})(r) & =k \underline{u}(r), \\
(\overline{k u})(r) & =k \bar{u}(r) .
\end{aligned}
$$

\section{Solving Coupled System of Matrix Equations Using Finite Iterative Algorithm [5]}

Matrix equations can be solved using various forms of the finite iterative algorithms example [1-3,5]. We consider iterative solutions to coupled system similar to the forms of Sylvester matrix equations [5].

$$
A V+B W=C,
$$

and second algorithm to solve coupled system of Sylvester matrix equations:

$$
\begin{aligned}
& A_{1} V+B_{1} W=C_{1}, \\
& A_{2} V+B_{2} W=C_{2} .
\end{aligned}
$$

Algorithm 1 (see [5]). A finite iterative algorithm is developed to solve equation (35) as follows:

(1) Input $A, B, C$.

(2) Pick arbitrary matrices $V \in \mathfrak{R}^{n \times p}$ and $W_{1} \in \mathfrak{R}^{r \times p}$.

(3) Set 


$$
\begin{aligned}
R_{1} & =C-A V_{1}-B W_{1}, \\
P_{1} & =A^{T} R_{1}, \\
Q_{1} & =B^{T} R_{1}, \\
K & =1 .
\end{aligned}
$$

(4) If $R_{K}=0$, then stop and $V_{K}$ and $W_{K}$ are the final solutions; else, let $K=K+1$ and go to step 5 .

(5) Calculate

$$
\begin{aligned}
V_{K+1} & =V_{K}+\frac{\left\|R_{K}\right\|^{2}}{\left\|P_{K}\right\|^{2}+\left\|Q_{K}\right\|^{2}} P_{K}, \\
W_{K+1} & =W_{K}+\frac{\left\|R_{K}\right\|^{2}}{\left\|P_{K}\right\|^{2}+\left\|Q_{K}\right\|^{2}} Q_{K}, \\
R_{K+1} & =C-A V_{K+1}-B W_{K+1} \\
& =R_{K}-\frac{\left\|R_{K}\right\|^{2}}{\left\|P_{K}\right\|^{2}+\left\|Q_{K}\right\|^{2}}\left|A P_{K}+B Q_{K}\right|, \\
P_{K+1} & =A^{T} R_{K+1}+\frac{\left\|R_{K+1}\right\|^{2}}{\left\|R_{K}\right\|^{2}} P_{K}, \\
Q_{K+1} & =B^{T} R_{K+1}+\frac{\left\|R_{K+1}\right\|^{2}}{\left\|R_{K}\right\|^{2}} Q_{K} .
\end{aligned}
$$

Algorithm 2 (see [5]). The following finite iterative algorithm is proposed to solve coupled system of Sylvester matrix equation (36):

(1) Input matrices: $A_{1} ; B_{1} ; A_{2} ; B_{2} ; C_{1} ; C_{2}$.

(2) Pick arbitrary matrices $Y_{1_{1}} \in C^{n \times p}$ and $Y_{2_{1}} \in C^{r \times p}$.

(3) Set

$$
\begin{aligned}
& R_{1}=\operatorname{diag}\left(C_{1}-f\left(Y_{1_{1}}, Y_{2_{1}}\right), C_{2}-g\left(Y_{1_{1}}, Y_{2_{1}}\right)\right), \\
& S_{1}=A_{1}^{T}\left(C_{1}-f\left(Y_{1_{1}}, Y_{2_{1}}\right)\right)+A_{2}^{T}\left(C_{2}-g\left(Y_{1_{1}}, Y_{2_{1}}\right)\right), \\
& T_{1}=B_{1}^{T}\left(C_{1}-f\left(Y_{1_{1}}, Y_{2_{1}}\right)\right)+B_{2}^{T}\left(C_{2}-g\left(Y_{1_{1}}, Y_{2_{1}}\right)\right) .
\end{aligned}
$$

(4) If $R_{K}=0$, then stop and $Y_{1_{K}}$ and $Y_{2_{K}}$ are the solutions; else set $K=K+1$ and then go to step 5 .

(5) Calculate

$$
\begin{aligned}
Y_{1_{K+1}} & =Y_{1_{K}}+\frac{\left\|R_{K}\right\|^{2}}{\left\|S_{K}\right\|^{2}+\left\|T_{K}\right\|^{2}} S_{K}, \\
Y_{2_{K+1}} & =Y_{2_{K}}+\frac{\left\|R_{K}\right\|^{2}}{\left\|S_{K}\right\|^{2}+\left\|T_{K}\right\|^{2}} T_{K}, \\
R_{K+1} & =\operatorname{diag}\left(C_{1}-f\left(Y_{1_{K+1}}, Y_{2_{K+1}}\right), C_{2}-g\left(Y_{1_{K+1}}, Y_{2_{K+1}}\right)\right) \\
& =R_{K}-\frac{\left\|R_{K}\right\|^{2}}{\left\|S_{K}\right\|^{2}+\left\|T_{K}\right\|^{2}} \operatorname{diag}\left(f\left(S_{K}, T_{K}\right), g\left(S_{K}, T_{K}\right)\right) \\
S_{K+1} & =A_{1}^{T}\left(C_{1}-f\left(Y_{1_{K+1}}, Y_{2_{K+1}}\right)\right)+A_{2}^{T}\left(C_{2}-g\left(Y_{1_{K+1}}, Y_{2_{K+1}}\right)\right)+\frac{\left\|R_{K+1}\right\|^{2}}{\left\|R_{K}\right\|^{2}} S_{K}, \\
T_{K+1} & =B_{1}^{T}\left(C_{1}-f\left(Y_{1_{K+1}}, Y_{2_{K+1}}\right)\right)+B_{2}^{T}\left(C_{2}-g\left(Y_{1_{K+1}}, Y_{2_{K+1}}\right)\right)+\frac{\left\|R_{K+1}\right\|^{2}}{\left\|R_{K}\right\|^{2}} T_{K} .
\end{aligned}
$$

\section{Two-Dimensional Fuzzy Fredholm Integral Equation}

Two-dimensional FIE of the second kind is defined as follows [24]:

$$
u(x, y)=f(x, y)+\lambda \int_{a}^{b} \int_{c}^{d} k(x, y, s, t) u(s, t) \mathrm{d} s \mathrm{~d} t
$$

The linear (2D-FFIE-2) is defined as

$$
\widetilde{u}(x, y)=\tilde{f}(x, y)+\lambda \int_{a}^{b} \int_{c}^{d}(x, y, s, t) \widetilde{u}(s, t) \mathrm{d} s \mathrm{~d} t,
$$

where $\tilde{u}(x, y)$ and $\tilde{f}(x, y)$ are fuzzy real functions on $S=[a, b] \times[c, d], k(x, y, s, t)$ is an arbitrary kernel function over $V=[a, b] \times[c, d] \times[a, b] \times[c, d]$, and $\widetilde{u}(x, y)$ is unknown on $S$. 
Throughout this paper, we consider 2D-FFIE-2 with $a=c=0, b=d=1$, and $\lambda=1$.

Now, introduce parametric form of a 2D-FFIE-2 with respect to Definition 3. Let $(\underline{f}(x, y, r), \bar{f}(x, y, r))$ and $(\underline{u}(x, y, r), \bar{u}(x, y, r)), \quad 0 \leq r \leq 1,(x, y) \in S$, be parametric form of $\tilde{f}(x, y)$ and $\tilde{u}(x, y)$, respectively. Then parametric form of $2 \mathrm{D}$-FFIE-2 is as follows:

$$
\begin{aligned}
& \underline{u}(x, y, r)=\underline{f}(x, y, r)+\iint_{0}^{1} v_{1}(x, y, s, t, \underline{u}(x, y, r), \bar{u}(x, y, r)) \mathrm{d} s \mathrm{~d} t, \\
& \bar{u}(x, y, r)=\bar{f}(x, y, r)+\iint_{0}^{1} v_{2}(x, y, s, t, \underline{u}(x, y, r), \bar{u}(x, y, r)) \mathrm{d} s \mathrm{~d} t, \\
& v_{1}(x, y, s, t, \underline{u}(x, y, r), \bar{u}(x, y, r))= \begin{cases}k(x, y, s, t) \underline{u}(s, t, r), & k(x, y, s, t) \geq 0, \\
k(x, y, s, t) \bar{u}(s, t, r), & k(x, y, s, t)<0,\end{cases} \\
& v_{1}(x, y, s, t, \underline{u}(x, y, r), \bar{u}(x, y, r))= \begin{cases}k(x, y, s, t) \bar{u} \underline{u}(s, t, r), & k(x, y, s, t) \geq 0, \\
k(x, y, s, t) \underline{u}(s, t, r), & k(x, y, s, t)<0,\end{cases}
\end{aligned}
$$

for each $0 \leq x, y \leq 1$ and $0 \leq r \leq 1$. We can see that equations (43) and (44) are system of Fredholm integral equation of the second kind with three variables in crisp case.

\section{Proposed Hybrid Iterative Technique}

6.1. Converting Linear Two-Dimensional FIEs of Second Kind to Two Crisp Coupled Systems. This section presents an efficient method for soling a $2 \mathrm{D}$-FFIE-2 by using $2 \mathrm{D}$-TFs.

First, consider the following equation:

$$
\widetilde{u}(x, y)=\tilde{f}(x, y)+\lambda \int_{a}^{b} \int_{c}^{d} k(x, y, s, t) \widetilde{u}(s, t) \mathrm{d} s \mathrm{~d} t .
$$

Now, the problem is to find the TF coefficients of $\tilde{u}(x, y)$ from the known functions $\widetilde{f}(x, y)$ and kernel $k(x, y, s, t)$. 2D-TFs are applied for equations

$$
\underline{u}(x, y, r)=\underline{f}(x, y, r)+\iint_{0}^{1} k(x, y, s, t) \underline{u}(s, t, r) \mathrm{d} s \mathrm{~d} t,
$$

$$
\bar{u}(x, y, r)=\bar{f}(x, y, r)+\iint_{0}^{1} k(x, y, s, t) \bar{u}(s, t, r) \mathrm{d} s \mathrm{~d} t .
$$

To describe the approach of equation (47), first expand $\underline{u}(x, y, r), f(x, y, r)$, and $k(x, y, s, t)$ by $2 \mathrm{D}$-TFs as follows:

$$
\begin{aligned}
\underline{u}(x, y, r) \simeq & T^{T}(x, y) U T(r), \\
\underline{u}(x, y, r) \simeq & T 11^{T}(x, y) U 11 T 1(r)+T 12^{T}(x, y) U 21 T 1(r)+T 21^{T}(x, y) U 31 T 1(r) \\
& +T 22^{T}(x, y) U 41 T 1(r)+T 11^{T}(x, y) U 12 T 2(r)+T 12^{T}(x, y) U 22 T 2(r) \\
& +T 21^{T}(x, y) U 32 T 2(r)+T 22^{T}(x, y) U 42 T 2(r), \\
\underline{f}(x, y, r) \simeq & T^{T}(x, y) F T(r), \\
\underline{f}(x, y, r) \simeq & T 11^{T}(x, y) F 11 T 1(r)+T 12^{T}(x, y) F 21 T 1(r) \\
& +T 21^{T}(x, y) F 31 T 1(r)+T 22^{T}(x, y) F 41 T 1(r)+T 11^{T}(x, y) F 12 T 2(r) \\
& +T 12^{T}(x, y) F 22 T 2(r)+T 21^{T}(x, y) F 32 T 2(r)+T 22^{T}(x, y) F 42 T 2(r), \\
k(x, y, s, t) \simeq & T \\
&
\end{aligned}
$$

where $T(x, y)$ and $T(r)$ are defined in equations (3) and (21), respectively, $U$ and $F$ are $\left(4 M^{2} \times 2 M\right)$ matrix of $2 \mathrm{D}-\mathrm{TF}$ coefficients of $\underline{u}(x, y, r)$ and $f(x, y, r)$, respectively, and $K$ is $\left(4 M^{2} \times 4 M^{2}\right)$-matrix 2D-TF coefficients of $(x, y, s, t)$. 
To obtain the solution of equation (47) from equations (49), (51), and (53), we have

$$
\begin{aligned}
& T^{T}(x, y) U T(r)=T^{T}(x, y) F T(r)+\iint_{0}^{1} T^{T}(x, y) K T(s, t) T^{T}(x, y) U T(r) \mathrm{d} s \mathrm{~d} t, \\
& T^{T}(x, y) U T(r)=T^{T}(x, y) F T(r)+T^{T}(x, y) K\left(\iint_{0}^{1} T^{T}(x, y) K T(s, t) T^{T}(x, y) \mathrm{d} s \mathrm{~d} t\right) U T(r) .
\end{aligned}
$$

Using equation (22), we have

$T^{T}(x, y) U T(r)=T^{T}(x, y) F T(r)+T^{T}(x, y) \operatorname{KDUT}(r)$,

and then

$$
U=F+K D U
$$

where $U$ and $F$ are $4 M^{2} \times 2 M$-matrix and $K D$ is $4 M^{2} \times 4 M^{2}$ - matrix, so $K D U$ is $4 M^{2} \times 2 M$-matrix, where $U$ is unknown.

Then, we have

$$
(I-K D) U=F,
$$

with

$$
\begin{aligned}
& U=\left[\begin{array}{ll}
U 11 & U 12 \\
U 21 & U 22 \\
U 31 & U 32 \\
U 41 & U 42
\end{array}\right], \\
& F=\left[\begin{array}{ll}
F 11 & F 12 \\
F 21 & F 22 \\
F 31 & F 32 \\
F 41 & F 42
\end{array}\right],
\end{aligned}
$$$$
K=\left[\begin{array}{llll}
K 11 & K 12 & K 13 & K 14 \\
K 21 & K 22 & K 23 & K 24 \\
K 31 & K 32 & K 33 & K 34 \\
K 41 & K 42 & K 43 & K 44
\end{array}\right]
$$$$
D=\left[\begin{array}{lllll}
\frac{h_{1}}{3} I_{1} \otimes \frac{h_{2}}{3} I_{2} & \frac{h_{1}}{3} I_{1} \otimes \frac{h_{2}}{6} I_{2} & \frac{h_{1}}{6} I_{1} \otimes \frac{h_{2}}{3} I_{2} & \frac{h_{1}}{6} I_{1} \otimes \frac{h_{2}}{6} I_{2} \\
\frac{h_{1}}{3} I_{1} \otimes \frac{h_{2}}{6} I_{2} & \frac{h_{1}}{3} I_{1} \otimes \frac{h_{2}}{3} I_{2} & \frac{h_{1}}{6} I_{1} \otimes \frac{h_{2}}{6} I_{2} & \frac{h_{1}}{6} I_{1} \otimes \frac{h_{2}}{3} I_{2} \\
\frac{h_{1}}{6} I_{1} \otimes \frac{h_{2}}{3} I_{2} & \frac{h_{1}}{6} I_{1} \otimes \frac{h_{2}}{6} I_{2} & h_{1} I_{1} \otimes \frac{h_{2}}{3} I_{2} & \frac{h_{1}}{3} I_{1} \otimes \frac{h_{2}}{6} I_{2} \\
\frac{h_{1}}{6} I_{1} \otimes \frac{h_{2}}{6} I_{2} & \frac{h_{1}}{6} I_{1} \otimes \frac{h_{2}}{3} I_{2} & h_{1} & \frac{h_{1}}{3} I_{1} \otimes \frac{h_{2}}{6} I_{2} & \frac{h_{1}}{3} I_{1} \otimes \frac{h_{2}}{3} I_{2}
\end{array}\right]
$$$$
I_{4 M^{2} \times 4 M^{2}}=\left[\begin{array}{cccc}
I_{M^{2}} & 0 & 0 & 0 \\
0 & I_{M^{2}} & 0 & 0 \\
0 & 0 & I_{M^{2}} & 0 \\
0 & 0 & 0 & I_{M^{2}}
\end{array}\right] \text {, }
$$

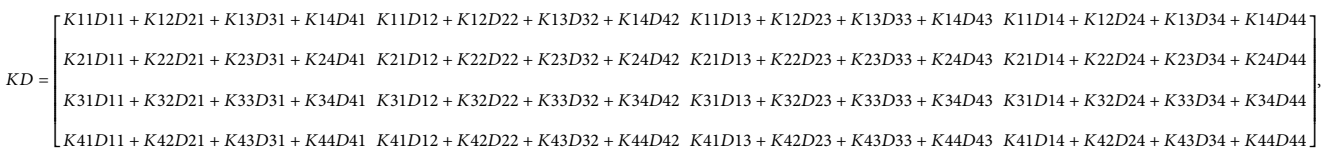

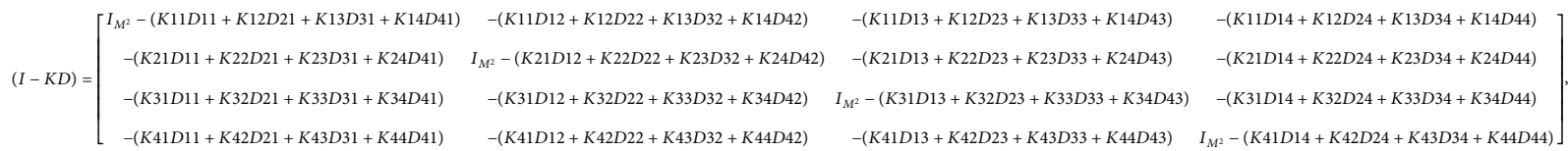
$(I-K D) U=F$

$\left(I_{M^{2}}-(K 11 D 11+K 12 D 21+K 13 D 31+K 14 D 41)\right) U 11-(K 11 D 12+K 12 D 22+K 13 D 32+K 14 D 42) U 21-(K 11 D 13+K 12 D 23+K 13 D 33+K 14 D 43) U 31-(K 11 D 14+K 12 D 24+K 13 D 34+K 14 D 44) U 41=F 11$, $-(K 21 D 11+K 22 D 21+K 23 D 31+K 24 D 41) U 11+I_{M^{2}}-(K 21 D 12+K 22 D 22+K 23 D 32+K 24 D 42) U 21-(K 21 D 13+K 22 D 23+K 23 D 33+K 24 D 43) U 31-(K 21 D 14+K 22 D 24+K 23 D 34+K 24 D 44) U 41=F 21$ $-(K 31 D 11+K 32 D 21+K 33 D 31+K 34 D 41) U 11-(K 31 D 12+K 32 D 22+K 33 D 32+K 34 D 42) U 21+\left(I_{M^{2}}-(K 31 D 13+K 32 D 23+K 33 D 33+K 34 D 43)\right) U 31-(K 31 D 14+K 32 D 24+K 33 D 34+K 34 D 44) U 41=F 31$, $-(K 41 D 11+K 42 D 21+K 43 D 31+K 44 D 41) U 11-(K 41 D 12+K 42 D 22+K 43 D 32+K 44 D 42) U 21-(K 41 D 13+K 42 D 23+K 43 D 33+K 44 D 43) U 31+\left(I_{M^{2}}-(K 41 D 14+K 42 D 24+K 43 D 34+K 44 D 44)\right) U 41=F 41$ 
Set

$A_{1}=I_{M^{2}}-(K 11 D 11+K 12 D 21+K 13 D 31+K 14 D 41)$,

$A_{2}=-(K 11 D 12+K 12 D 22+K 13 D 32+K 14 D 42)$,

$A_{3}=-(K 11 D 13+K 12 D 23+K 13 D 33+K 14 D 43)$,

$A_{4}=-(K 11 D 14+K 12 D 24+K 13 D 34+K 14 D 44)$,

$B_{1}=-(K 21 D 11+K 22 D 21+K 23 D 31+K 24 D 41)$,

$B_{2}=I_{M^{2}}-(K 21 D 12+K 22 D 22+K 23 D 32+K 24 D 42)$,

$B_{3}=-(K 21 D 13+K 22 D 23+K 23 D 33+K 24 D 43)$,

$B_{4}=-(K 21 D 14+K 22 D 24+K 23 D 34+K 24 D 44)$,

$C_{1}=-(K 31 D 11+K 32 D 21+K 33 D 31+K 34 D 41)$,

$C_{2}=-(K 31 D 12+K 32 D 22+K 33 D 32+K 34 D 42)$,

$D_{1}=-(K 41 D 11+K 42 D 21+K 43 D 31+K 44 D 41)$,

$D_{2}=-(K 41 D 12+K 42 D 22+K 43 D 32+K 44 D 42)$,

$D_{3}=-(K 41 D 13+K 42 D 23+K 43 D 33+K 44 D 43)$,

$D_{4}=I_{M^{2}}-(K 41 D 14+K 42 D 24+K 43 D 34+K 44 D 44)$,

which lead to the following two crisp linear systems:

$$
\begin{aligned}
& A_{1} U 11+A_{2} U 21+A_{3} U 31+A_{4} U 41=F 11, \\
& B_{1} U 11+B_{2} U 21+B_{3} U 31+B_{4} U 41=F 21, \\
& C_{1} U 11+C_{2} U 21+C_{3} U 31+C_{4} U 41=F 31, \\
& D_{1} U 11+D_{2} U 21+D_{3} U 31+D_{4} U 41=F 41,
\end{aligned}
$$

and

$$
A_{1} U 12+A_{2} U 22+A_{3} U 32+A_{4} U 42=F 12,
$$

$$
\begin{aligned}
& B_{1} U 12+B_{2} U 22+B_{3} U 32+B_{4} U 42=F 22, \\
& C_{1} U 12+C_{2} U 22+C_{3} U 32+C_{4} U 42=F 32, \\
& D_{1} U 12+D_{2} U 22+D_{3} U 32+D_{4} U 42=F 42 .
\end{aligned}
$$

Similarly, we expand $\bar{u}(x, y, r)$ and $\bar{f}(x, y, r)$ by 2D-TFs, and by substituting them into equation (42), two coupled crisp linear systems, similar to (61) and (65), are obtained. It is clear that all matrices in the two coupled crisp linear systems (61) and (65) are square matrices of dimensions $M \times M$. Thus, we need to obtain the coefficient matrices $U 11, U 12, U 21, U 22, U 31, U 32, U 41$, and $U 42$ in order to get the approximate numerical solution of the form:

$$
\underline{u}(x, y, r)=T^{T}(x, y) U T(r) .
$$

6.2. Proposed Iterative Algorithm for Solving Coupled Systems (61) and (65). An iterative algorithm is proposed here to solve the two coupled systems (61) and (65) as a generalization of Algorithm 2.

Algorithm 3. Algorithm 2 is modified and generalized to work out for systems (61) and (65) as follows.

First, for coupled system (61):

(1) Input $A_{1} ; A_{2} ; A_{3} ; A_{4} ; B_{1} ; B_{2} ; B_{3} ; B_{4} ; C_{1} ; C_{2} ; C_{3} ; C_{4}$; $D_{1} ; D_{2} ; D_{3} ; D_{4} ; F 11 ; F 21 ; F 31 ; F 41$.

(2) Choose arbitrary matrices $U 11, U 21, U 31, U 41$.

(3) For $k=1$, set

$$
\begin{aligned}
R_{k}= & \operatorname{diag}(F 11-f 1(U 11, U 21, U 31, U 41), F 21-f 2(U 11, U 21, U 31, U 41), F 31-f 3(U 11, U 21, U 31, U 41), F 41 \\
& -f 4(U 11, U 21, U 31, U 41)) \\
S 1_{k}= & A_{1}^{T}(F 11-f 1(U 11, U 21, U 31, U 41))+B_{1}{ }^{T}(F 21-f 2(U 11, U 21, U 31, U 41))+C_{1}{ }^{T}(F 31-f 3(U 11, U 21, U 31, U 41)) \\
& +D_{1}{ }^{T}(F 41-f 4(U 11, U 21, U 31, U 41)), \\
S 2_{k}= & A_{2}^{T}(F 11-f 1(U 11, U 21, U 31, U 41))+B_{2}{ }^{T}(F 21-f 2(U 11, U 21, U 31, U 41))+C_{2}{ }^{T}(F 31-f 3(U 11, U 21, U 31, U 41)) \\
& +D_{2}{ }^{T}(F 41-f 4(U 11, U 21, U 31, U 41)), \\
S 3_{k}= & A_{3}^{T}(F 11-f 1(U 11, U 21, U 31, U 41))+B_{3}{ }^{T}(F 21-f 2(U 11, U 21, U 31, U 41))+C_{3}{ }^{T}(F 31-f 3(U 11, U 21, U 31, U 41)) \\
& +D_{3}{ }^{T}(F 41-f 4(U 11, U 21, U 31, U 41)), \\
S 4_{k}= & A_{4}^{T}(F 11-f 1(U 11, U 21, U 31, U 41))+B_{4}{ }^{T}(F 21-f 2(U 11, U 21, U 31, U 41))+C_{4}{ }^{T}(F 31-f 3(U 11, U 21, U 31, U 41)) \\
& +D_{4}{ }^{T}(F 41-f 4(U 11, U 21, U 31, U 41)) .
\end{aligned}
$$


(4) If $R_{K}=0$, then stop and $U 11, U 21, U 31, U 41$ are the solutions; else, let $K=K+1$ and go to step 5 .

(5) Compute

$$
\begin{aligned}
& U 11=U 11+\frac{\left\|R_{K}\right\|^{2}}{\left\|S 1_{k}\right\|^{2}+\left\|S 2_{k}\right\|^{2}+\left\|S 3_{k}\right\|^{2}+\left\|S 4_{k}\right\|^{2}} S 1_{k} \\
& U 21=U 21+\frac{\left\|R_{K}\right\|^{2}}{\left\|S 1_{k}\right\|^{2}+\left\|S 2_{k}\right\|^{2}+\left\|S 3_{k}\right\|^{2}+\left\|S 4_{k}\right\|^{2}} S 2_{k}, \\
& U 31=U 31+\frac{\left\|R_{K}\right\|^{2}}{\left\|S 1_{k}\right\|^{2}+\left\|S 2_{k}\right\|^{2}+\left\|S 3_{k}\right\|^{2}+\left\|S 4_{k}\right\|^{2}} S 3_{k}, \\
& U 41=U 41+\frac{\left\|R_{K}\right\|^{2}}{\left\|S 1_{k}\right\|^{2}+\left\|S 2_{k}\right\|^{2}+\left\|S 3_{k}\right\|^{2}+\left\|S 4_{k}\right\|^{2}} S 4_{k}, \\
& R_{k+1}=\operatorname{diag}(F 11-f 1(U 11, U 21, U 31, U 41), F 21-f 2(U 11, U 21, U 31, U 41), F 31-f 3(U 11, U 21, U 31, U 41), \\
& \text { - } F 41-f 4(U 11, U 21, U 31, U 41)) \text {, } \\
& S 1_{k+1}=A_{1}^{T}(F 11-f 1(U 11, U 21, U 31, U 41))+B_{1}^{T}(F 21-f 2(U 11, U 21, U 31, U 41))+C_{1}^{T}(F 31-f 3(U 11, U 21, U 31, U 41)) \\
& +D_{1}^{T}(F 41-f 4(U 11, U 21, U 31, U 41))+\frac{\left\|R_{K+1}\right\|^{2}}{\left\|R_{K}\right\|^{2}} S 1_{K}, \\
& S 2_{k+1}=A_{2}^{T}(F 11-f 1(U 11, U 21, U 31, U 41))+B_{2}^{T}(F 21-f 2(U 11, U 21, U 31, U 41))+C_{2}^{T}(F 31-f 3(U 11, U 21, U 31, U 41)) \\
& +D_{2}^{T}(F 41-f 4(U 11, U 21, U 31, U 41))+\frac{\left\|R_{K+1}\right\|^{2}}{\left\|R_{K}\right\|^{2}} S 2_{K}, \\
& S 3_{k+1}=A_{3}^{T}(F 11-f 1(U 11, U 21, U 31, U 41))+B_{3}^{T}(F 21-f 2(U 11, U 21, U 31, U 41))+C_{3}^{T}(F 31-f 3(U 11, U 21, U 31, U 41)) \\
& +D_{3}^{T}(F 41-f 4(U 11, U 21, U 31, U 41))+\frac{\left\|R_{K+1}\right\|^{2}}{\left\|R_{K}\right\|^{2}} S 3_{K}, \\
& S 4_{k+1}=A_{4}^{T}(F 11-f 1(U 11, U 21, U 31, U 41))+B_{4}^{T}(F 21-f 2(U 11, U 21, U 31, U 41))+C_{4}^{T}(F 31-f 3(U 11, U 21, U 31, U 41)) \\
& +D_{4}^{T}(F 41-f 4(U 11, U 21, U 31, U 41))+\frac{\left\|R_{K+1}\right\|^{2}}{\left\|R_{K}\right\|^{2}} S 4_{K} .
\end{aligned}
$$

For coupled system (65), the algorithm is repeated with replacing $U 11, U 21, U 31, U 41$ by $U 12, U 22, U 32, U 42$ and $F 11, F 21, F 31, F 41$ by $F 12, F 22, F 32, F 42$ where the $2 M \times$ $2 M$ block $U$ matrix is computed.
The approximate crisp numerical solution for equation (51) of the form $\underline{u}(x, r)_{\text {approx. }}=T^{T}(x) U T(r)$ is then obtained.

In a similar manner, the crisp numerical solution for equation (53) of the form $\bar{u}(x, r)_{\text {approx. }}=T^{T}(x) U T(r)$ can 
be obtained by carrying out the above-proposed algorithm for the other coupled crisp systems similar to (61) and (65).
Finally, the solution for linear 2D-FFIE-2 by 2D-TFs is then given as

$$
u(x, y)=\left(\underline{u}_{\text {approx }}(x, y, r), \bar{u}_{\text {approx }}(x, y, r)\right), \quad 0 \leq r \leq 1, x, y \in[0,1)
$$

\section{Convergence Analysis of the Proposed Method}

In this section, we obtain error estimate for the numerical method proposed in previous section.

Theorem 1. The solution of the two-dimensional Fredholm fuzzy integral equations given by equation (1) by using $2 D$ TFs converges to exact solution if

$$
S=\max _{0 \leq x, y, s, t \leq 1}|k(x, y, s, t)|<1
$$

Proof. Assume $\tilde{u}_{\text {exact }}(x, y)$ and $\tilde{u}_{\text {approx }}(x, y)$ represent the exact and approximate solutions of equation (1), respectively. Therefore,

$$
\begin{aligned}
\tilde{u}_{\text {approx }}(x, y)= & \sum_{i=0}^{m_{1}-1} \sum_{j=0}^{m_{2}-1} c_{i, j} T_{i, j}^{1,1}(s, t)+\sum_{i=0}^{m_{1}-1} \sum_{j=0}^{m_{2}-1} d_{i, j} T_{i, j}^{1,2}(s, t) \\
& +\sum_{i=0}^{m_{1}-1} \sum_{j=0}^{m_{2}-1} e_{i, j} T_{i, j}^{2,1}(s, t)+\sum_{i=0}^{m_{1}-1} \sum_{j=0}^{m_{2}-1} l_{i, j} T_{i, j}^{2,2}(s, t) .
\end{aligned}
$$

By using equation (1), we can write

$$
\begin{aligned}
e(x, y)= & \left\|\tilde{u}_{\text {exact }}(x, y)-\tilde{u}_{\text {approx }}(x, y)\right\|=\max _{(x, y) \in[0,1]}\left|\tilde{u}_{\text {exact }}(x, y)-\tilde{u}_{\text {approx }}(x, y)\right| \\
= & \max _{(x, y) \in[0,1]} \mid\left(\int_{0}^{1} \int_{0}^{1} k(x, y, s, t) \widetilde{u}_{\text {exact }}(s, t) \mathrm{d} s \mathrm{~d} t-\int_{0}^{1} \int_{0}^{1} k(x, y, s, t)\left(\sum_{i=0}^{m_{1}-1} \sum_{j=0}^{m_{2}-1} c_{i, j} T_{i, j}^{1,1}(s, t)+\sum_{i=0}^{m_{1}-1} \sum_{j=0}^{m_{2}-1} d_{i, j} T_{i, j}^{1,2}(s, t)\right.\right. \\
& \left.\left.+\sum_{i=0}^{m_{1}-1} \sum_{j=0}^{m_{2}-1} e_{i, j} T_{i, j}^{2,1}(s, t)+\sum_{i=0}^{m_{1}-1} \sum_{j=0}^{m_{2}-1} l_{i, j} T_{i, j}^{2,2}(s, t)\right) \mathrm{d} s \mathrm{~d} t\right) \mid \\
\leq & S\left(\int_{0}^{1} \int_{0}^{1} \max _{(x, y) \in[0,1]}\left|\tilde{u}_{\text {exact }}(s, t)-\tilde{u}_{\text {approx }}(s, t)\right| \mathrm{d} s \mathrm{~d} t\right)=S\left(\int_{0}^{1} \int_{0}^{1}\left\|\widetilde{u}_{\text {exact }}(s, t)-\widetilde{u}_{\text {approx }}(s, t)\right\| \mathrm{d} s \mathrm{~d} t\right)
\end{aligned}
$$

where

$$
S=\max _{0 \leq x, y, s, t \leq 1}|k(x, y, s, t)|<\infty .
$$

Also, we have $\lim _{M \rightarrow \infty} \tilde{u}_{\text {approx }}(x, y)=\tilde{u}_{\text {exact }}(x, y)$, so $\left\|\widetilde{u}_{\text {exact }}(x, y)-\widetilde{u}_{\text {approx }}(x, y)\right\| \longrightarrow 0$ as $M \longrightarrow \infty$ and since $S$ is bounded.

Thus,

$$
\lim _{M \longrightarrow \infty}\left\|\widetilde{u}_{\text {exact }}(x, y)-\widetilde{u}_{\text {approx }}(x, y)\right\| \longrightarrow 0,
$$

so the proof of the theorem is completed.

Remark 1. In our theoretical investigation for the proposed method, we take $m 1=m 2=M$.

\section{Numerical Results and Discussion}

This section demonstrates the effectiveness and the accuracy of our proposed hybrid method, 2D-TFs and an iterative algorithm, on some examples. The solution of each example is obtained for different values of $x, y, r$, and $M$ and is compared with the exact solution, the direct method, and the presented method when the tolerance criteria residual is $>e^{-4}$ and $>e^{-8}$.

Example 1. The 2D-FFIE-2 given in Mirzaee et al. [15] is considered as

$$
\begin{aligned}
& \underline{f}(x, y, r)=r\left(x y+\frac{1}{676}\left(x^{2}+y^{2}-2\right)\right), \\
& \bar{f}(x, y, r)=(2-r)\left(x y+\frac{1}{676}\left(x^{2}+y^{2}-1\right)\right), \\
& k(x, y, s, t)=\frac{1}{169}\left(x^{2}+y^{2}-2\right)\left(s^{2}+t^{2}-2\right), \quad 0 \leq x, t \leq 1 \text { and } \lambda=1 .
\end{aligned}
$$

In this case, the exact solution is given by

$$
\widetilde{u}(x, y, r)=(\underline{u}(x, y, r), \bar{u}(x, y, r))=(r x y,(2-r) x y) .
$$

The number of iterations for solving the two coupled matrix equations to obtain the coefficient matrices taken by our proposed iterative algorithm is $k=3$ when the tolerance criteria residual is $>e^{-4}$ which indicates that the hybrid 
TABLe 1: Numerical results with TF system for Example 1 for $x=0.1, y=0.4$, and $M=4$.

\begin{tabular}{|c|c|c|c|c|c|}
\hline$r$ & Exact solution $\underline{u}(x, y, r)$ & Direct method [15] & Absolute error & Presented method & Absolute error \\
\hline 0 & 0.00000000 & 0.00000000 & $8.4015 e-006$ & 0.00000002 & $1.89187044 e-008$ \\
\hline 0.1 & 0.00400000 & 0.00398678 & $1.3220 e-005$ & 0.00399416 & $5.83560842 e-006$ \\
\hline 0.2 & 0.00800000 & 0.00797356 & $2.6440 e-005$ & 0.00798831 & $1.16901355 e-005$ \\
\hline 0.3 & 0.01200000 & 0.01196034 & $3.9660 e-005$ & 0.01198246 & $1.75446627 e-005$ \\
\hline 0.4 & 0.01600000 & 0.01594712 & $5.2880 e-005$ & 0.01597660 & $2.33991898 e-005$ \\
\hline 0.5 & 0.02000000 & 0.01993390 & $6.6100 e-005$ & 0.01997075 & $2.92537169 e-005$ \\
\hline 0.6 & 0.02400000 & 0.02392068 & $7.9320 e-005$ & 0.02396489 & $3.51082441 e-005$ \\
\hline 0.7 & 0.02800000 & 0.02700746 & $9.9254 e-004$ & 0.02796099 & $4.09627712 e-005$ \\
\hline 0.8 & 0.03200000 & 0.03189424 & $1.0576 e-004$ & 0.03195318 & $4.68172983 e-005$ \\
\hline 0.9 & 0.03600000 & 0.03588102 & $1.1898 e-004$ & 0.03594733 & $5.26718254 e-005$ \\
\hline
\end{tabular}

TABle 2: Numerical results with TF system for Example 1 for $x=0.1, y=0.4$, and $M=4$.

\begin{tabular}{|c|c|c|c|c|c|}
\hline$r$ & Exact solution $\bar{u}(x, y, r)$ & Direct method [15] & Absolute error & Presented method & Absolute error \\
\hline 0 & 0.08000000 & 0.07973560 & $2.6440 e-004$ & 0.07988291 & $1.17085832 e-004$ \\
\hline 0.1 & 0.07600000 & 0.07574882 & $2.5118 e-004$ & 0.07588878 & $1.11222414 e-004$ \\
\hline 0.2 & 0.07200000 & 0.07176204 & $2.3796 e-004$ & 0.07189464 & $1.05358995 e-004$ \\
\hline 0.3 & 0.06800000 & 0.06777526 & $2.2474 e-004$ & 0.06790050 & $9.94955768 e-005$ \\
\hline 0.4 & 0.06400000 & 0.06378849 & $2.1151 e-004$ & 0.06390637 & $9.36321584 e-005$ \\
\hline 0.5 & 0.06000000 & 0.05980170 & $1.9830 e-004$ & 0.05991223 & $8.77687400 e-005$ \\
\hline 0.6 & 0.05600000 & 0.05581492 & $1.8508 e-004$ & 0.05592806 & $8.19053216 e-005$ \\
\hline 0.7 & 0.05200000 & 0.05182814 & $1.7186 e-004$ & 0.05192396 & $7.60419032 e-005$ \\
\hline 0.8 & 0.04800000 & 0.04784136 & $1.5864 e-004$ & 0.04792982 & $7.01784848 e-005$ \\
\hline 0.9 & 0.04400000 & 0.04385458 & $1.4542 e-004$ & 0.04393568 & $6.43150664 e-005$ \\
\hline
\end{tabular}

proposed method is quite efficient and has good accuracy as seen from Tables 1 and 2 .

Remark 2. The numerical results for the approximate solution using the direct method in Tables 1 and 2 are taken from Table 3 in [15], while the numerical results using the direct and the proposed iterative methods in Tables 3 and 4 are obtained using our own program written using MATLAB R2018b. Also, the number of iterations for solving the two coupled matrix equations to obtain the coefficient matrices taken by our proposed iterative algorithm is $k=3$ when the tolerance criteria residual is $>e^{-4}$ which indicates that the hybrid proposed method is quite efficient and has good accuracy as seen from Tables 3 and 4 .

Example 2. Consider the following made up linear twodimensional fuzzy Fredholm integral equations (2D-FFIE2):

$$
\begin{aligned}
\underline{f}(x, y, r) & =\frac{5}{12} r(x+y), \\
\bar{f}(x, y, r) & =\frac{5}{12}(2-r)(x+y), \\
k(x, y, s, t) & =x s+y t, \quad 0 \leq x, t \leq 1 \text { and } \lambda=1 .
\end{aligned}
$$

$$
\widetilde{u}(x, y, r)=(\underline{u}(x, y, r), \bar{u}(x, y, r))=(r(x+y),(2-r)(x+y)) .
$$

Remark 3. The number of iterations for solving the two coupled matrix equations to obtain the coefficient matrices taken by our proposed iterative algorithm is $k=3$ when the tolerance criteria residual is $>e^{-4}$ which indicates that the hybrid proposed method is quite efficient and has good accuracy as seen from Tables 5 and 6 .

Example 3. Consider the following 2D-FFIE-2:

$$
\begin{aligned}
\underline{f}(x, y, r) & =\frac{11}{36} r x^{2} y^{2}, \\
\bar{f}(x, r) & =\frac{11}{36}(2-r) x^{2} y^{2}, \\
k(x, y, s, t) & =x^{2} y^{2}(1+2 t)(1+2 s), \quad 0 \leq x, t \leq 1 \text { and } \lambda=1 .
\end{aligned}
$$

In this case, the exact solution is given by

$$
\begin{aligned}
\underline{u}(x, y, r) & =r x^{2} y^{2}, \\
\bar{u}(x, r) & =(2-r) x^{2} y^{2} .
\end{aligned}
$$

In this case, the exact solution is given by 
TABLe 3: Numerical results with TF system for Example 1 for $x=0.1, y=0.4$, and $M=6$.

\begin{tabular}{lcccrc}
\hline$r$ & Exact solution $\underline{u}(x, y, r)$ & Direct method & Absolute error & Presented method & Absolute error \\
\hline 0 & 0.00000000 & 0.00000239 & $2.39402667 e-006$ & 0.00000010 & $9.77129847 e-008$ \\
0.1 & 0.00400000 & 0.00399511 & $4.89026667 e-006$ & 0.00399758 & $2.42072449 e-006$ \\
0.2 & 0.00800000 & 0.00799261 & $7.38650667 e-006$ & 0.00799506 & $4.93916197 e-006$ \\
0.3 & 0.01200000 & 0.01199012 & $9.88274667 e-006$ & 0.01199254 & $7.45759945 e-006$ \\
0.4 & 0.01600000 & 0.01598762 & $1.23789867 e-005$ & 0.01599002 & $9.97603693 e-006$ \\
0.5 & 0.02000000 & 0.01998512 & $1.48752267 e-005$ & 0.01998751 & $1.24944744 e-005$ \\
0.6 & 0.02400000 & 0.02398263 & $1.73714667 e-005$ & 0.02398499 & $1.50129119 e-005$ \\
0.7 & 0.02800000 & 0.02798013 & $1.98677067 e-005$ & 0.02798247 & $1.75313494 e-005$ \\
0.8 & 0.03200000 & 0.03197764 & $2.23639467 e-005$ & 0.03197995 & $2.00497868 e-005$ \\
0.9 & 0.03600000 & 0.03597514 & $2.48601867 e-005$ & 0.03597743 & $2.25682243 e-005$ \\
\hline
\end{tabular}

TABle 4: Numerical results with TF system for Example 1 for $x=0.1, y=0.4$, and $M=6$.

\begin{tabular}{|c|c|c|c|c|c|}
\hline$r$ & Exact solution $\bar{u}(x, y, r)$ & Direct method & Absolute error & Presented method & Absolute error \\
\hline 0 & 0.08000000 & 0.07994709 & $5.29077333 e-005$ & 0.07994927 & $5.07341947 e-005$ \\
\hline 0.1 & 0.07600000 & 0.07594863 & $5.13736533 e-005$ & 0.07595198 & $4.80210603 e-005$ \\
\hline 0.2 & 0.07200000 & 0.07195016 & $4.98395733 e-005$ & 0.07195469 & $4.53079259 e-005$ \\
\hline 0.3 & 0.06800000 & 0.06795169 & $4.83054933 e-005$ & 0.06795741 & $4.25947915 e-005$ \\
\hline 0.4 & 0.06400000 & 0.06395323 & $4.67714133 e-005$ & 0.06396012 & $3.98816571 e-005$ \\
\hline 0.5 & 0.06000000 & 0.05995476 & $4.52373333 e-005$ & 0.05996283 & $3.71685227 e-005$ \\
\hline 0.6 & 0.05600000 & 0.05595630 & $4.37032533 e-005$ & 0.05596554 & $3.44553883 e-005$ \\
\hline 0.7 & 0.05200000 & 0.05195783 & $4.21691733 e-005$ & 0.05196826 & $3.17422539 e-005$ \\
\hline 0.8 & 0.04800000 & 0.04795936 & $4.06350933 e-005$ & 0.04797097 & $2.90291195 e-005$ \\
\hline 0.9 & 0.04400000 & 0.04396090 & $3.91010133 e-005$ & 0.04397368 & $2.63159851 e-005$ \\
\hline
\end{tabular}

TABLe 5: Numerical results with TF system for Example 2 for $x=0.1, y=0.1$, and $M=4$.

\begin{tabular}{lcccccc}
\hline$r$ & Exact solution $\underline{u}(x, y, r)$ & Presented method & Absolute error & Exact solution $\bar{u}(x, y, r)$ & Presented method & Absolute error \\
\hline 0 & 0.00000000 & 0.00000000 & $1.734723476 e-17$ & 0.40000000 & 0.40000000 & $0.000000000 e+00$ \\
0.1 & 0.02000000 & 0.02000000 & $1.387778781 e-17$ & 0.38000000 & 0.38000000 & $0.000000000 e+00$ \\
0.2 & 0.04000000 & 0.04000000 & $1.387778781 e-17$ & 0.36000000 & 0.36000000 & $5.551115123 e-17$ \\
0.3 & 0.06000000 & 0.06000000 & $0.000000000 e+00$ & 0.34000000 & 0.34000000 & $0.000000000 e+00$ \\
0.4 & 0.08000000 & 0.08000000 & $0.000000000 e+00$ & 0.32000000 & 0.32000000 & $0.000000000 e+00$ \\
0.5 & 0.10000000 & 0.10000000 & $0.000000000 e+00$ & 0.30000000 & 0.30000000 & $0.000000000 e+00$ \\
0.6 & 0.12000000 & 0.12000000 & $2.775557562 e-17$ & 0.28000000 & 0.28000000 & $5.551115123 e-17$ \\
0.7 & 0.14000000 & 0.14000000 & $2.775557562 e-17$ & 0.26000000 & 0.26000000 & $5.551115123 e-17$ \\
0.8 & 0.16000000 & 0.16000000 & $0.000000000 e+00$ & 0.24000000 & 0.24000000 & $2.775557562 e-17$ \\
0.9 & 0.18000000 & 0.18000000 & $1.734723476 e-17$ & 0.22000000 & 0.22000000 & $5.551115123 e-17$ \\
\hline
\end{tabular}

TABLE 6: Numerical results with TF system for Example 2 for $x=0.1, y=0.1$, and $M=6$.

\begin{tabular}{lcccccc}
\hline$r$ & Exact solution $\underline{u}(x, y, r)$ & Presented method & Absolute error & Exact solution $\bar{u}(x, y, r)$ & Presented method & Absolute error \\
\hline 0 & 0.00000000 & 0.00000000 & $0.000000000 e+00$ & 0.40000000 & 0.40000000 & $0.000000000 e+00$ \\
0.1 & 0.02000000 & 0.02000000 & $0.000000000 e+00$ & 0.38000000 & 0.38000000 & $0.000000000 e+00$ \\
0.2 & 0.04000000 & 0.04000000 & $0.000000000 e+00$ & 0.36000000 & 0.36000000 & $0.000000000 e+00$ \\
0.3 & 0.06000000 & 0.06000000 & $0.000000000 e+00$ & 0.34000000 & 0.34000000 & $0.000000000 e+00$ \\
0.4 & 0.08000000 & 0.08000000 & $0.000000000 e+00$ & 0.32000000 & 0.32000000 & $0.000000000 e+00$ \\
0.5 & 0.10000000 & 0.10000000 & $0.000000000 e+00$ & 0.30000000 & 0.30000000 & $0.000000000 e+00$ \\
0.6 & 0.12000000 & 0.12000000 & $0.000000000 e+00$ & 0.28000000 & 0.28000000 & $0.000000000 e+00$ \\
0.7 & 0.14000000 & 0.14000000 & $0.000000000 e+00$ & 0.26000000 & 0.26000000 & $0.000000000 e+00$ \\
0.8 & 0.16000000 & 0.16000000 & $0.000000000 e+00$ & 0.24000000 & 0.24000000 & $0.000000000 e+00$ \\
0.9 & 0.18000000 & 0.18000000 & $0.000000000 e+00$ & 0.22000000 & 0.22000000 & $0.000000000 e+00$ \\
\hline
\end{tabular}


TABLE 7: Numerical results with TF system for Example 3 for $x=0.25, y=0.25$, and $M=6$.

\begin{tabular}{lcccccc}
\hline$r$ & Exact solution $\underline{u}(x, y, r)$ & Presented method & Absolute error & Exact solution $\bar{u}(x, y, r)$ & Presented method & Absolute error \\
\hline 0 & 0.00000000 & 0.00000232 & $2.32197716 e-006$ & 0.00781250 & 0.00609664 & $1.71585866 e-003$ \\
0.1 & 0.00039063 & 0.00030680 & $8.38225958 e-005$ & 0.00742187 & 0.00579187 & $1.63000792 e-003$ \\
0.2 & 0.00078125 & 0.00061128 & $1.69967169 e-004$ & 0.00703125 & 0.00548709 & $1.54415718 e-003$ \\
0.3 & 0.00117188 & 0.00091576 & $2.56111742 e-004$ & 0.00664062 & 0.00518232 & $1.45830644 e-003$ \\
0.4 & 0.00156250 & 0.00122024 & $3.42256315 e-004$ & 0.00625000 & 0.00487754 & $1.37245569 e-003$ \\
0.5 & 0.00195313 & 0.00152472 & $4.28400888 e-004$ & 0.00585938 & 0.00457277 & $1.28660495 e-003$ \\
0.6 & 0.00234375 & 0.00182920 & $5.14545461 e-004$ & 0.00546875 & 0.00426800 & $1.20075421 e-003$ \\
0.7 & 0.00273437 & 0.00213368 & $6.00690034 e-004$ & 0.00507813 & 0.00396322 & $1.11490347 e-003$ \\
0.8 & 0.00312500 & 0.00243817 & $6.86834607 e-004$ & 0.00468750 & 0.00365845 & $1.02905273 e-003$ \\
0.9 & 0.00351562 & 0.00274265 & $7.72979179 e-004$ & 0.00429688 & 0.00335367 & $9.43201983 e-004$ \\
\hline
\end{tabular}

Remark 4. In Table 7, the number of iterations for this example by our proposed iterative algorithm is $k=3$ when the tolerance criteria residual is $>e^{-4}$ which indicates that the hybrid proposed method is quite efficient. Moreover, we can see that our method has good accuracy which can be further improved by increasing the residual.

\section{Conclusion}

Fuzzy control applications and a large proportion of applied mathematical topics require the solution of the fuzzy integral equations. The paper introduced the 2D-TFs method for approximating the solution of linear 2D-FFIE-2, which is a hybrid of triangular functions and an iterative algorithm. The method is simple, efficient, and accurate and is based on converting the original equation into two crisp systems (2DFFIE-2). The efficiency and simplicity of the proposed method are demonstrated via numerical examples with known exact solutions, and the results are given. Furthermore, the exceptional value of the proposed method is low cost of the equation setting with no need for any projection method or integration.

\section{Data Availability}

The data used to support the findings of this study are available from the corresponding author upon request.

\section{Conflicts of Interest}

The authors declare that they have no conflicts of interest.

\section{Authors' Contributions}

The authors declare that the study was realized in collaboration with the same responsibility. All authors read and approved the manuscript.

\section{Acknowledgments}

The authors did not receive any funds or grants from any public or private institutions.

\section{References}

[1] K. E. Atkinson, The Numerical Solution of Integral Equations of the Second Kind, Cambridge University Press, Cambridge, UK, 1997.
[2] L. M. Delves and J. L. Mohamed, Computational Methods for Integral Equation, Cambridge University Press, Cambridge, UK, 1985.

[3] M. C. Bonis and C. Laurita, "Numerical treatment of second kind Fredholm integral equations systems on bounded interval," Journal of Computational and Applied Mathematics, vol. 217, pp. 67-87, 2008.

[4] K. Maleknejad, R. Mollapourasl, and M. Alizadeh, "Numerical solution of Volterra type integral equation of the first kind with wavelet basis," Applied Mathematics and Computation, vol. 194, no. 2, pp. 400-405, 2007.

[5] K. Maleknejad and F. Mirzaee, "Numerical solution of linear Fredholm integral equations system by rationalized Haar functions method," International Journal of Computer Mathematics, vol. 80, no. 11, pp. 1397-1405, 2003.

[6] E. Babolian, H. R. Marzban, and M. Salmani, "Using triangular orthogonal functions for solving Fredholm integral equations of the second kind," Applied Mathematics and Computation, vol. 201, no. 12, pp. 452-464, 2008.

[7] M. A. Ramadan and M. R. Ali, "An efficient hybrid method for solving Fredholm integral equations using triangular functions," New Trends in Mathematical Sciences, vol. 5, no. 1, pp. 213-224, 2017.

[8] M. Ramadan, H. Oshaba, and R. Kharabsheh, "Triangular functions based method for the solution of system of linear Fredholm integral equations via an efficient finite iterative algorithm," Journal of Intelligent \& Fuzzy Systems, vol. 38, no. 3, pp. 2847-2858, 2020.

[9] K. Maleknejad, M. Shahrezaee, and H. Khatami, "Numerical solution of integral equations system of the second kind by block pulse functions," Applied Mathematics and Computation, vol. 166, no. 1, pp. 15-24, 2005.

[10] M. Jahantigh, T. Allahviranloo, and M. Otadi, "Numerical solution of fuzzy integral equation," Applied Mathematical Sciences, vol. 2, pp. 33-46, 2008.

[11] F. Mirzaee, M. Paripour, and M. Komak Yari, "Numerical solution of Fredholm fuzzy integral equations of the second kind via direct method using triangular functions," Journal of Hyperstructures, vol. 1, no. 2, pp. 46-60, 2012.

[12] M. Ghanbari, M. R. Toushmalni, and E. Kamrani, "Numerical solution of linear Fredholm fuzzy integral equation of the second kind by block-pulse functions," Australian Journal of Basic and Applied Sciences, vol. 3, no. 3, pp. 2637-2642, 2009.

[13] M. Barkhordary, N. A. Kiani, and A. R. Bozorgmanesh, "A method for solving fuzzy Fredholm integral equations of the second kind," International Center For Scientific Research and Studies, vol. 1, no. 2, 2008.

[14] M. Ramadan, T. S. El-Danaf, A. R. Hadhoud, and H. S. Osheba, "An accurate and efficient technique for approximating fuzzy Fredholm integral equations of the second 
kind using triangular functions," New Trends in Mathematical Sciences, vol. 8, no. 2, pp. 29-43, 2020.

[15] F. Mirzaee, M. K. Yari, and E. Hadadiyan, "Numerical solution of two-dimensional fuzzy Fredholm integral equations of the second kind using triangular functions," Beni-Suef University Journal of Basic and Applied Sciences, vol. 4, no. 2, pp. 109-118, 2015.

[16] H. Nouriani and R. Ezzati, "Quadrature iterative method for numerical solution of two-dimensional linear fuzzy Fredholm integral equations," Mathematical Sciences, vol. 11, no. 1, pp. 63-72, 2017.

[17] R. Ezzati and S. Ziari, "Numerical solution of two-dimensional fuzzy Fredholm integral equations of the second kind using fuzzy bivariate Bernstein polynomials," International Journal of Fuzzy Systems, vol. 15, no. 1, pp. 84-89, 2013.

[18] E. Hengamian Asl and J. Saberi-Nadjafi, "Triangular functions with convergence for solving linear system of two-dimensional fuzzy Fredholm integral equation," Jordan Journal of Mathematics and Statistics (JJMS), vol. 12, no. 4, pp. 557$580,2019$.

[19] A. M. Bica and C. Popescu, "Fuzzy trapezoidal cubature rule and application to two-dimensional fuzzy Fredholm integral equations," Soft Computing, vol. 21, no. 5, pp. 1229-1243, 2017.

[20] A. Rivaz and F. Yousefi, "Modified homotopy perturbation method for solving two-dimensional fuzzy Fredholm integral equation," International Journal of Applied Mathematics, vol. 25, no. 4, pp. 591-602, 2012.

[21] A. Georgieva, "Solving two-dimensional nonlinear VolterraFredholm fuzzy integral equations by using Adomian decomposition method," Dynamic Systems and Applications, vol. 27, no. 4, pp. 819-835, 2018.

[22] S. Karamseraji, R. Ezzati, and S. Ziari, "Fuzzy bivariate triangular functions with application to nonlinear fuzzy Fredholm-Volterra integral equations in two dimensions," Soft Computing, vol. 24, no. 12, pp. 9091-9103, 2019.

[23] M. A. Ramadan, M. A. Abdel Naby, T. S. El-Danaf, and A. M. E. Bayoumi, "Finite iterative algorithm for solving a class of complex matrix equation with two unknowns of general form," Applied and Computational Mathematics, vol. 3, no. 5, pp. 273-284, 2014.

[24] W.-J. Xie and F.-R. Lin, "A fast numerical solution method for two dimensional Fredholm integral equations of the second kind," Applied Numerical Mathematics, vol. 59, no. 7, pp. 1709-1719, 2009. 